\title{
Philosophiques
}

\section{L’Université et la crise " comptable »}

\section{Georges A. Legault}

Volume 9, numéro 2, octobre 1982

URI : https://id.erudit.org/iderudit/203199ar

DOI : https://doi.org/10.7202/203199ar

Aller au sommaire du numéro

Éditeur(s)

Société de philosophie du Québec

ISSN

0316-2923 (imprimé)

1492-1391 (numérique)

Découvrir la revue

Citer ce document

Legault, G. A. (1982). L’Université et la crise « comptable ». Philosophiques, 9(2), 317-336. https://doi.org/10.7202/203199ar d'utilisation que vous pouvez consulter en ligne.

https://apropos.erudit.org/fr/usagers/politique-dutilisation/ 


\title{
L'UNIVERSITÉ ET LA CRISE «COMPTABLE»
}

\author{
par Georges A. Legault
}

Certains mots, comme les vêtements, sont de mise dans certains contextes. Étiquettes commodes, signifiant tout et rien de précis, ils prétendent résumer une situation. Au Québec, dans les conversations quotidiennes comme dans les discours politiques, le mot crise tisse de nombreux propos. Chargé affectivement par son usage historique, «la grande crise», ce mot non seulement progresse en intensité avec la fréquence élevée de son utilisation mais encore en extension, puisqu'on l'applique pour qualifier de nombreuses situations. Qu'est-ce qui n'est pas «en crise»? $\grave{A}$ première vue, les propos de $M$. le ministre Laurin, de février 81 , semblent exclure l'université de la crise.

Au seuil des années 80 , cette mission éducative et culturelle des universités est toujours, et peut-être plus que jamais, opportune et nécessaire. Au moment où les diverses crises que traversent les pays industrialisés (crise de l'économie, crise de l'énergie, crise du partage du pouvoir, crise des valeurs, crise des identités, etc.) prennent peu à peu l'allure d'une «crise de culture et de civilisation», la réalisation de la mission éducative et culturelle des universités revêt tous les traits d'une tâche urgente et prioritaire.

Mais la suite nous convainc du contraire: l'université n'échappe pas à la crise.

Des universités - ces lieux de créativité, d'imagination, d'innovation et de réflexion critique - ne sommes-nous pas fondés d'attendre des éléments de réponse, l'identification des visions et des pratiques qui permettront de garder l'avenir ouvert? ${ }^{1}$

L'université n'échappe pas à la crise parce que d'une part, on attend quelque chose d'elle et cette attente est fondée et d'autre

1. Docteur Camille Laurin. L'Avenir des universités québécoises: vers une politique des universités. Discours prononcé devant les représentants de la communauté universitaire québécoise, à l'Université de Montréal, le 3 février 1981. Gouvernement du Québec, Ministère de l'éducation, p.4. 
part, parce que les universités ne répondent pas à ces attentes légitimes. L'université doit donc rendre des comptes: faire le bilan, expliquer, justifier mais aussi, se rendre compte de ce qu'elle ne fait pas.

Depuis ce discours, la remise en cause de l'institution universitaire s'est manifestée en paroles (que l'on songe aux nombreuses déclarations concernant la charge de travail des professeurs) et en acte ( les compressions budgétaires imposées et annoncées pour l'avenir). Il ne s'agit pas ici d'excuser l'université, de l'exonérer de toute critique ou de justifier les privilèges de ses membres, mais de voir comment le débat sur les universités s'est orienté depuis peu, comment il traduit un conflit de société et un changement de mentalité qui aboutiront à une réforme du réseau universitaire.

On peut mesurer l'évolution du débat sur les universités par l'emphase graduelle de la comptabilisation de l'activité universitaire. Cette notion, empruntée au modèle économique, véhicule un aspect de mesure, de bilan financier, (crédit-débit), de responsabilité (en dette), suppose aussi une personne à qui on est redevable (contrôleur) et à qui revient des décisions (actionnaire, bailleur de fonds). La rhétorique concernant les universités, gravite autour de ces thèmes et justifie de nouvelles approches décisionnelles.

\section{1. ÉVOLUTION DE LA «COMPTABILISATION»DE L'ACTIVITÉ} UNIVERSITAIRE

À écouter les nombreux discours sur l'université dans la presse écrite et parlée, on peut s'étonner de voir l'ampleur de la remise en question de l'université. Il n'y a pas si longtemps, comme le rappelait Marc Laurendeau, animateur du débat sur L'Avenir de l'université tenu à Montréal le 4 mai 1978, on valorisait l'éducation et on était prêt à investir, sans soulever d'objections, dans les institutions universitaires. Des universités de qualité comparables à celles de nos voisins de l'ouest et du sud étaient une priorité. Si les étudiants d'alors contestaient la qualité de certains cours ou programmes, la valeur éducative, culturelle et scientifique de l'université n'était point contestée.

Mais en 1978, le climat était différent. Le 29 juin 1977 un arrêté-en-conseil avait décidé la création d'une Commission d'étude 
sur les universités avec le mandat "d'effectuer une étude publique sur l'avenir de l'enseignement supérieur et des universités du Québec». Et en mai 1978, le Gouvernement du Québec annonçait une coupure de 13 millions de dollars dans les budgets des universités pour l'exercice financier de 1978-1979. Dès ce moment, les pôles du débat actuel sur les univesités étaient tracés. Ces pôles structurent encore les discussions d'aujourd'hui: les décisions gouvernementales en matière de coupures budgétaires, la réflexion critique sur l'université et la défense de l'université comme valeur traditionnelle.

Nos présents débats s'articulent à partir de ces pôles, mais les champs de force ont été modifiés depuis 1978. La Commission d'étude a produit ses rapports ${ }^{2}$ et le 7 mars 1980 , le Conseil des universités présentait son avis sur les trois premiers rapports de la Commission ${ }^{3}$. Pour sa part, le Gouvernement du Québec annonçait des coupures de 80 millions de dollars dans le réseau universitaire, les justifiant par le contexte économique difficile qui s'abat sur les québécois. L'argumentation économique et les accusations de "gaspillage» ont vite pris l'avant-scène du débat dans la presse. Certains professeurs d'université et la FAPUQ ont répliqué dans les journaux à ces reproches ou semi-vérités. À la fin de l'année 1981, le débat a changé de direction: les interventions de certains professeurs d'universités et du Conseil supérieur de l'éducation l'ont porté à un autre niveau en s'interrogeant sur le caractère même des coupures budgétaires. Dans sa recommandation au ministre de l'éducation, adoptée en novembre 1981, le Conseil supérieur de l'éducation affirme: . . . «L'enseignement supérieur, pour sa part, et malgré les conclusions d'une vaste Commission d'étude, ne dispose, encore aujourd'hui, pour tout éclairage, que d'intentions ambigües: il faut en faire lecture à travers le casse-

2. Commission d'étude sur les universités.

Comité de coordination, Rapport; Gouvernement du Québec, mai 1979.

Comité d'étude sur l'université et la société québécoise, Rapport, Gouvernement du Québec, mai 1979.

Comité d'étude sur la formation et le perfectionnement des enseignants, Rapport, Gouvernement du Québec, mai 1971.

Comité d'étude sur l'organisation du système universitaire, Rapport, Parties 1, 2, 3 , Gouvernement du Québec, mai 1979.

3. Conseil des universités, L'université québécoise des années 80 , Avis.du Conseil des universités sur les trois rapports de la Commission d'étude sur les universités, Gouvernement du Québec, mars 80. 
tête budgétaire dont on ne sait trop s'il est «conjoncturel»ou «structurant». ${ }^{4}$

En s'interrogeant ainsi à propos du caractère des compressions budgétaires, le Conseil supérieur de l'éducation offrait une nouvelle perspective sur le problème en cause: d'une part, il rattachait l'analyse comptable des budgets des universités à l'analyse structurelle qu'avaient fournie la Commission d'étude sur les universités et le Conseil des universités; et d'autre part, il rattachait l'analyse structurelle à la critique de l'université traditionnelle. C'est pourquoi le Conseil supérieur de l'éducation publiait, en même temps que son avis ${ }^{4}$, un document sur le rôle de l'universités. En soulevant ce doute sur les politiques gouvernementales, le Conseil supérieur suggère que dans l'hypothèse où les coupures ne sont pas uniquement justifiables par la conjoncture économique, elles constituent un mode d'intervention indirect dans l'orientation des universités et en poursuivent ainsi la réforme.

On comprend davantage la position du Conseil supérieur lorsqu'on la replace dans l'ensemble des débats sur l'université. Les avis contraires du Conseil supérieur de l'éducation et du Conseil des universités réflètent les positions divergentes adoptées par les Comités d'étude sur les universités. Ces divergences s'expriment d'abord, généralement, sur la valeur de l'université, mais plus spécifiquement sur son «accountability». Cette notion joue un rôle clef dans l'orientation des débats sur l'université: par elle, la valeur de l'université traditionnelle est remise en cause à plusieurs niveaux. Le terme «accountability» est utilisé tel quel dans le rapport du Comité d'étude sur l'université et la société québécoise, puisqu'il n'existe pas d'équivalent français ${ }^{6}$. Sous cette notion, traitée au chapitre $\mathrm{V}$ intitulé: «S'assurer que les universités assument leurs responsabilités envers la société» nous retrouvons l'idée de rentabilité de l'enseignement supérieur ${ }^{7}$ et celle de la productivité des universités ${ }^{8}$.

4. Conseil supérieur de l'éducation, Les compressions budgétaires et l'avenir des universités, Recommandation au ministre de l'éducation, adoptée à la $256^{\mathrm{e}}$ réunion du Conseil, novembre 1981.

5. Conseil supérieur de l'éducation, Le rôle spécifique de l'université, 10, 12, 81.

6. Commission d'étude sur les universités, Comité d'étude sur l'université et la société québécoise, Rapport, p. 65.

7. Ibid., p. 67.

8. Ibid., p. 69. 
Le problème de la rentabilité de l'enseignement supérieur est posé essentiellement en termes d'investissement de fonds publics dans l'éducation supérieure. «Est-ce trop? Trop peu en comparaison avec d'autres sociétés?» Le comité soulève seulement la complexité de cette question et s'interroge sur la rentabilité économique du réseau suite aux augmentations des dépenses depuis quinze ans.

Le comité analyse la productivité des universités avec la même attitude. Indiquant seulement des pistes en vue du développement d'indicateurs, faute d'outils suffisants, le Comité propose:

1) Le respect de l'efficience: les universités doivent accomplir leurs fonctions au meilleur coût;

2) l'aspect efficacité (impact): les universités doivent jouer leur rôle le mieux possible, produire des résultats appropriés aux besoins et aux attentes;

3) la productivité démontrée et la rentabilité des investissements: les universités doivent produire une quantité et une qualité de bénéfices dont la valeur égale ou excède la valeur des ressources utilisées pour les produire;

4) la responsabilité sociale: la performance des universités doit tenir compte des notions prévalentes de justice et d'équité9.

Les recommandations du Comité sur cette question traduisent ce «souci d'accountability" présenté dans le chapitre V. Ces recommandations portent sur un meilleur dialogue, la publication de rapports, la mise en place d'un système d'informations stables, la circulation de l'information et la mise en place d'indicateurs de performance et de productivité.

L'avis du Conseil des universités sur les trois rapports de la Commission accentue l'importance du modèle de l'accountability. La conception de l'université qui y est véhiculée s'inspire d'une démarche de planification institutionnelle pour l'ensemble du réseau universitaire et ceci pour chaque activité d'enseignement, de recherche ou de service à la collectivité. Du modèle d'accountability défini dans le rapport du Comité d'étude sur l'université et la société, l'avis retient l'aspect efficacité (impact). Il ne s'agit pas d'évaluer le rendement d'un individu mais la

9. Ibid., p. 69. 
pertinence même de l'activité. Ainsi un professeur peut accomplir des tâches d'enseignement, de recherche ou de service à la collectivité de manière très efficace mais cela ne garantit pas que son activité soit jugée pertinente. L'enseignement de telle matière est-il nécessaire à ce programme aùjourd'hui? Ce programme est-il nécessaire à la faculté? Cette faculté est-elle encore nécessaire à l'université? Cette université est-elle nécessaire au réseau québécois?

La pensée qui sous-tend l'évaluation de l'impact de l'activité dans la société d'aujourd'hui s'articule en termes d'objectifs. Chaque activité doit viser un objectif précis, ce qui permet l'évaluation continue de l'activité. C'est ce que propose en définitive le Conseil des universités:

- retient, en conclusion, que toute définition de l'université ne peut avoir de valeur que dans la mesure où elle introduit à une vision opérante des objectifs devant présider à l'orientation, à l'organisation, à l'évaluation des activités; croit donc que c'est dans le cadre d'une démarche itérative axée sur un plan d'action que peut le mieux prendre corps la réflexion sur le développement des universités...10

Une telle conception vise essentiellement l'augmentation de la qualité du produit fini, à l'étudiant, les résultats de la recherche ou les services à la collectivité. Pour atteindre des résultats acceptables, il faudrait définir:

1. en général, les objectifs des universités;

2. les objectifs spécifiques de chaque université dans le réseau;

3. les objectifs de chaque faculté, module, etc.;

4. les objectifs de chaque programme;

5. les objectifs de chaque cours du programme.

On devrait faire la même opération pour la recherche et les services à la collectivité. Si la définition des objectifs est une opération, l'évaluation des objectifs en est une autre. Chaque objectif devra passer le test de la pertinence en fonction de l'étudiant, du savoir ou de la collectivité.

10. Conseil des universités, op. cit., p. 34. 
La finalité première de ce modèle demeure l'évaluation de l'efficacité par la qualité des résultats obtenus. Les économies qu'on pourrait réaliser, par suite de cette opération, résulteraient d'une analyse de la qualité et découleraient de choix sociaux, de valeurs reconnues par les principaux partenaires de l'enseignement supérieur.

L'accountability, ainsi comprise par le Conseil des universités, modifie le schème d'argumentation traditionnel. Alors que tous semblaient d'accord sur la valeur de l'université comme institution à mission éducative et culturelle, le nouveau modèle met en cause la valeur de l'université et la rend "comptabilisable». Il s'agit donc, par le biais des objectifs, de trouver de nouveaux accords sur la valeur de l'université. Ce modèle s'oppose au modèle traditionnel de ce point de vue; par contre, il s'en rapproche dans la mesure où l'accord sur la valeur de l'université, une fois réalisé, implique nécessairement un investissement financier suffisant. Le débat porte donc sur la définition de l'université, et non pas sur les sommes qu'on y investit.

Cependant, l'accountability a plusieurs facettes, comme le souligne le rapport sur l'université et la société: l'aspect efficacité n'est qu'une dimension car il existe encore l'aspect de l'efficience et celui de la rentabilité de l'investissement. C'est par ce biais que le débat actuel sur les universités du Québec a été inscrit dans un débat plus vaste, celui de la crise économique.

Le problème économique du Québec est avant tout un problème de budget, de dette publique. Comme le soulignait, M. Pierre Fortin, économiste de l'Université Laval, dans un article publié le 14 janvier 1982 dans Le Devoir:

Résumons. Au rythme où vont les choses, le déficit budgétaire du Québec aura triplé et la dette publique aura quadruplé entre 1980 et 1985 . Les conséquences économiques et politiques de cette évolution, si elle devait se produire, seraient incalculables et tragiques. Les causes de la détresse financière du gouvernement ne résident pas dans un niveau trop bas des impôts et dans la conjoncture économique mauvaise, mais principalement dans l'élan de croissance apparamment irrépressible des dépenses budgétaires et, secondairement seulement, dans les coupures de transferts fédéraux. La croissance plus rapide qu'ailleurs du niveau des services publics offerts, mais dépend dans une très large mesure du nombre trop élevé des employés rémunérés par unité de service 
rendu et des conditions de travail exorbitantes accordées aux fonctionnaires par les deux dernières conventions collectives (Bourassa et Parizeau).

Pour réduire les investissements dans les services sociaux, on peut en contester l'efficience ou la rentabilité (la productivité). L'université n'a pas échappé à cette double critique. Les premiers reproches qui lui furent adressés visaient son efficience. En effet, les coûts élevés des universités au Québec sont expliqués par, la faible charge de travail des professeurs ( 6 heures semaine), les salaires élevés, les dédoublements, etc. Après, on a reproché aux universités leur rapport coûts-bénéfices, c'est-à-dire, la faiblesse de leur rentabilité et de leur productivité.

Le glissement du modèle d'accountability prôné par le Conseil des universités au modèle comptable, apparaît déjà dans la conférence du ministre Laurin sur les universités.

Dans une telle conjoncture, il faudra nécessairement que nous acceptions de soulever la question de ce que des analystes et des citoyens de plus en plus nombreux appellent la "productivité» des ressources et des activités universitaires. (Je n'aime pas beaucoup recourir à ce mot, à cause de connotations industrielles et commerciales, voire mercantiles, qui décrivent plutôt mal le caractère fondamentalement éducatif et culturel des retombées qu'on est en droit d'attendre des pratiques universitaires. Parlons alors plutôt de "fécondité» ou même, si vou le voulez bien, de "productivité» — à condition qu'on s'entende bien sur la nature propre des résultats des activités universitaires et sur l'incongruité qu'il y aurait à se livrer ici à quelque comptabilisation simpliste de la rentabilité). Nous chercherons donc à savoir si nous tirons tout le parti possible de nos ressources. Il faudra même accepter de nous demander si nous ne pourrions pas accroître la "productivité» ou la «fécondité» de nos équipements, de nos appareils d'administration et de gestion aussi bien que nos activités d'enseignement, de recherche et de services à la collectivité. Ces analyses devront-elles aller jusqu'à remettre en cause certaines façons de définir et d'évaluer les tâches d'enseignement, de recherche et de gestion? ${ }^{11}$

Malgré certaines réserves quant aux difficultés à mesurer la productivité ou la fécondité, le ministre concluait tout de même qu'une analyse de la productivité pourrait permettre de statuer sur la fermeture de certains programmes.

11. Docteur Camille Laurin, op. cit., p. 5. 
Le contexte des coupures budgétaires allait provoquer, dans les institutions, la recherche de formules de productivité. L'expression "productivité» associée, au début, avec «fécondité», a rapidement perdu cette connotation qualitative pour n'exprimer que le rapport coût/services. Dans ce modèle, il s'agit essentiellement de mesurer le coût de la pièce usinée. Toutes les questions sur la qualité du produit ou des performances sont éliminées du débat. Il s'agit d'établir des rapports «objectifs» «neutres» facilement vérifiables.

Pour atteindre cet objectif, nous pouvons adopter l'analyse du produit fini (output) ou celle de la matière première (input). La première situation nous conduit à comparer le rapport coûts/nombre de diplômés du 1er, 2ème et 3ème cycle. Les produits semi-finis sont comptabilisés comme pure perte. La deuxième opération privilégie le rapport coûts/nombre d'étudiants. Cependant, pour que cette méthode soit opérationnelle, il faut «ventiler» les chiffres, et par conséquent s'entendre sur les unités de base servant à estimer les coûts. Pour qu'elle unité de base doit-on établir le rapport coûts/nombre d'étudiants? Pour chaque tâche d'enseignement, pour chaque professeur, pour chaque département, pour chaque faculté ou module? Comment doit-on y inclure les coûts des administrations, des services auxiliaires et de l'immobilisation? Des questions analogues se posent lorsqu'on veut déterminer la variable «étudiants». S'agit-il des étudiants d'un cours, d'un département, d'un programme, d'une faculté ou d'un module?

Plusieurs formules peuvent donc être générées à partir de la même situation et chacune d'elle donnera un résultat différent. Peu importe la formule utilisée, il s'agit toujours de la même constante: rapport coûts/services. À Sherbrooke, par exemple, on a établi un indicateur de productivité représentant le nombre de crédits-étudiants produits par crédit d'enseignement dispensé et on a calculé que la productivité moyenne pour notre université s'établissait à 0.80 . Il s'agit donc d'une moyenne inférieure à la productivité retrouvée dans les autres universités du Québec. Dans cette formule, les éléments de comparaison sont les créditsétudiants et les crédits d'enseignement dispensés. De telles formules permettent des comparaisons quantitatives au prix d'une abstraction de l'enseignement réel, dans des contextes géographi- 
ques distincts. Par exemple, cette formule réduit le travail du professeur à l'enseignement et élimine l'encadrement. Aucune dimension qualitative n'est retenue. Évidemment, des cours tutoraux qui sont donnés en plus de la charge d'enseignement d'un professeur, sont considérés comme activité d'enseignement au même titre que les autres et ont, parce qu'il s'agit d'un tutoral, un indice de productivité quasiment nul. En se basant sur de telles formules pour fermer des cours, des programmes ou pour trouver d'autres solutions, on cherchera toujours à augmenter la quantité. C'est la seule dimension que l'analyse comptable peut mettre en évidence.

Les formules coûts/étudiants peuvent nous donner des indications, nous décrire selon différents paramètres une partie des facteurs relativement aux coûts des services. Cependant, ces descriptions ne sont que des descriptions. Partant de leur analyse, on ne peut pas inférer directement un jugement de valeur. Pour passer de ce qui est à ce qui doit être, il faut au moins une prémisse impliquant des valeurs. Ainsi, deux individus, devant des chiffres fournis par des formules de productivité, pourraient avoir des réactions contraires. Si les bas coûts sont seuls importants (jugement de valeur), une personne pourra louer les programmes à haute productivité. Par contre, si la qualité de la formation (jugement de valeur), est plus déterminante que les coûts, une personne pourra être fière du bas taux de productivité fourni par la formule. Dans le modèle comptable, il y a un jugement implicite, les bas coûts sont une valeur.

Le modèle comptable n'est pas seulement appliqué à l'enseignement. On essaie aussi d'élaborer des formules pour mesurer la productivité de la recherche subventionnée et non subventionnée. Dans le domaine des recherches subventionnées, le principal critère retenu est le montant des subventions, leur fréquence. Pour les autres recherches, on tente de quantifier, par le biais des publications, la productivité de la recherche.

Ce modèle de la rentabilité économique des investissements, avec le contrôle minitieux de l'efficience et de la productivité, modifie le cadre traditionnel des débats sur l'université, et ceci à plusieurs points de vue: celui des accords pré-supposés à toute argumentation, celui des données retenues enfin celui du contexte psycho-social des accords. Au niveau des accords, le 
débat actuel intègre toutes les discussions sur l'université à celles qui portent sur l'ensemble des services sociaux par le biais de l'investissement des fonds publics. Il ne s'agit pas de discuter des valeurs des différentes institutions et de fixer des priorités sociales comme le supposait l'ancien modèle, mais de considérer la capacité de payer des contribuables comme limite des valeurs sociales inscrites dans les institutions publiques. L'appel aux contribuables devient alors le critère des choix sociaux et des choix relatifs aux montants des investissements. Le débat sur les valeurs est dès lors subsumé sous la notion de rentabilité. On détermine la meilleure rentabilité par la comparaison avec nos voisins, à l'ouest ou au sud, en supposant que la qualité est toujours égale. On retrouve un exemple de cette rhétorique, dans l'article déjà cité de M. Pierre Fortin.

Dans les écoles primaires et secondaires on rémunère un enseignant par 16 élèves, au lieu d'un par 21 élèves comme en Ontario. Le nombre d'enseignants québécois est donc «trop élevé» de $30 \%$ par rapport à l'Ontario... Nos enfants savent-ils pour autant mieux lire, écrire et compter que les enfants ontariens? ${ }^{12}$

D'un certain point de vue, la prédominance du modèle comptable dans les débats actuels sur l'université semble refléter l'absence de consensus, d'accord sur les valeurs sociales dans notre société. Parmi toutes les valeurs possibles, et également défendables, comment justifier des choix prioritaires et établir une hiérarchie des valeurs? Et puisque toutes ces valeurs sociales dépendent des investissements, qui se réflètent dans les tables d'imposition, n'est-il pas dès lors «normal» de poser le problème en terme d'investissement?

D'un autre point de vue, la prédominance de ce modèle comptable semble réfléter, par le biais des contrôles qu'il implique, un autre malaise de notre société, à savoir la méfiance ou l'absence systématique de confiance. Chaque modèle de l'université dépend, pour son fonctionnement, de la confiance qu'on accorde aux différents intervenants. Par exemple, la Commission d'étude sur les universités et le Conseil des universités proposent deux conceptions divergentes de l'université. En fait, le rapport du Comité de coordination de la Commission d'étude définit

12. Pierre Fortin, «Les finances publiques, un coup de barre radical s'impose», Le Devoir, 14 janvier 1982. 
l'université comme une «entreprise à visée essentiellement éducative et culturelle» ${ }^{13}$. Cette mission de l'université n'est pas conçue comme une abstraction, une idée générale et vague mais comme un principe qui oriente et anime la vie universitaire.

... le principe évoque une manière d'être créant un climat et
inspirant un style de vie et de pensée qui imprègne toute l'activité
universitaire et lui confere une tonalité propre. Il s'agit en d'au-
tres termes d'un ensemble de valeurs, d'attitudes et de comporte-
ments qui constitue une tradition vivante, enracinée dans le passé
et ouverte sur un avenir qu'elle est capable d'inventer, voire
parfois de modeler sous diverses formes ${ }^{14}$.

Une telle conception de l'université dépend étroitement de la confiance accordée aux professeurs. Le professeur est libre de choisir ses priorités et d'établir les tâches qu'il se donne à l'intérieur de l'enseignement et de l'encadrement, de la recherche ou des services à la collectivité. Aucun modèle standard n'est applicable pour toute la collectivité universitaire d'une université et de l'ensemble du réseau. De plus, d'année en année, le professeur peut modifier son programme personnel selon ses priorités.

Dans ce modèle, il n'y a aucun contrôle systématique de l'efficacité ou de la performance du professeur. Certes, l'évaluation joue un rôle dans l'obtention de la permanence après 3 ou 5 ans ou dans l'octroi des promotions. Cependant, ces évaluations visent davantage à déterminer si on peut faire confiance au candidat. C'est pourquoi, après le rang de professeur titulaire, il n'existe aucune autre évaluation des activités du professeur.

Tant que la confiance règne, ce modèle peut être efficace. Mais à partir du moment où cette confiance accordée aux individus et à l'institution ne semble pas garantir l'accomplissement des objectifs de l'université, on exige dès lors que les institutions se fixent des objectifs précis. Par ce moyen, on espère pouvoir évaluer périodiquement l'atteinte des objectifs et contrôler les activités. La conception de l'université adoptée par le Conseil des universités véhicule cette démarche de planification institutionnelle pour l'ensemble du réseau universitaire et pour chaque activité d'enseignement, de recherche et de service à la collectivité. Et après avoir résumé la conception de l'université présentée

13. Commission d'étude sur les universités, Comité de coordination, Rapport, p. 12.

14. Ibid., p. 12. 
par la Commission d'étude sur les universités, le Conseil en déclare explicitement les motifs: "On peut penser qu'il est nécessaire, dans toutes les matières concernées, d'aller plus loin que le simple rappel aux bonnes volontés, et de miser sur autre chose qu'une seule stratégie de croissance de fonds: ce sont là précisément les postulats ayant été au fondement des politiques de naguère, et qui méritent d'être attentivement reconsidérés, au vu des résultats qu'ils ont engendrés» ${ }^{15}$.

Mais cette crise de confiance peut aller plus loin, elle peut s'adresser aux individus et aux associations syndicales. Lorsque le gouvernement lança sa campagne pour «couper le gras», il a reproché aux universitaires leur faible charge de travail ( 6 heures par semaine). Cet argument joue plusieurs rôles puisqu'il sert de base de comparaison avec les professeurs des autres régions en même tant qu'il montre l'écart apparamment injustifié entre le salaire et la charge de travail. De plus, cet argument se veut descriptif, non pas des libellés des conventions collectives mais de la pratique réelle des professeurs. Ainsi, on soupçonne les professeurs de ne rien faire: peu d'enseignement, aucun encadrement (les étudiants se plaignent), peu de recherche ( $20 \%$ seulement des professeurs) et perte de temps dans des réunions et des services à la collectivité non définis. Derrière l'expression «couper dans le gras» se cache un jugement sur les individus et les institutions qui se sont «engraissées» au dépens des autres.

\section{ATTITUDES DÉCISIONNELLES}

L'évolution du débat sur les universités, depuis l'acceptation incontestée de sa valeur jusqu'à la crise comptable, est parallèle à une autre, celle du rapport entre le discours et la prise de décision. Le Conseil des universités et le Conseil supérieur de l'éducation malgré leur divergence de vues concernant la conception de l'université, réclament tous les deux dans leurs avis respectifs un débat public sur l'ensemble de la question universitaire. On suppose ici qu'un débat entre les parties concernées par la chose universitaire aboutira à des solutions justifiées, acceptées, et ainsi à des réformes concrètes. L'approche comptable modifie cette perspective puisqu'elle met en scène, comme principal intéressé,

15. Conseil des universités, op. cit., p. 134-135. 
les investisseurs, les payeurs de taxe. Le débat sur les universités est public parce qu'il se tient sur la place publique, en termes d'investissements et de rentabilité. Il n'est pas étonnant de constater, dès lors, que le seul document officiel concernant une politique des universités soit la conférence prononcée par $\mathbf{M}$. le ministre Laurin il y a plus d'un an. La voie du silence, de l'écoute pratiquée depuis, même aux ateliers du ministre, se conjugue aux déclarations publiques sur le gaspillage et aux mesures de compressions budgétaires imposées dans les universités.

L'opinion publique sert de moyen de pression à deux niveaux. Elle est utilisée pour justifier les compressions budgétaires et pour discréditer toute rhétorique adverse qui n'irait pas dans le sens des compressions: «éliminer le gras» d'abord, "faire plus avec moins» ensuite. De ce fait, l'argument de la productivité permet d'implanter une réforme de l'enseignement universitaire sous le regard approbateur de l'opinion publique.

L'argument de la productivité joue un rôle clé dans la conférence du ministre Laurin, car il devient le critère premier pour évaluer les différentes tâches qui composent généralement la charge de travail d'un universitaire: services à la collectivité, administration, recherche et enseignement-encadrement. La structure de l'argument est simple. Chaque fonction de la charge de travail est soumise au critère de productivité et un bilan négatif exige son retrait ou sa réforme.

Ainsi, dans sa conférence du 3 février 1981, monsieur le ministre affirme:

En fait, tout indique que, sur cette question pourtant fort engageante, les débats réels n'ont pas encore vraiment eu lieu dans l'ensemble des universités. On peut même dire que nous ne disposons pas d'un tableau complet et fidèle de ce qui se fait sous cette rubrique des "services à la collectivité».

Il est opportun et nécessaire que, en concertation avec les autres ressources éducatives et culturelles de leur région d'appartenance, les universités fassent le point sur cette question et précisent leurs positions institutionnelles. Il devrait sans doute résulter certains émondages, peut-être aussi certaines nouvelles zones d'expérimentation. De son côté, et à la lumière de ce débat, le ministère s'emploiera à définir les conditions et les modalités d'un financement judicieux et équitable de ces activités ${ }^{16}$.

16. Docteur Camille Laurin, op. cit., p. 10. 
Certes les services à la communauté sont difficile à définir, du moins à formuler de manière exhaustive. Plusieurs services paraissent davantage liés à la fonction de parents, d'autres au travail professionnel. Les premiers ne devraient pas être comptabilisés dans la charge de travail, les autres ne devraient pas être rénumérés. Entre le bénévolat et le double salaire, il semble y avoir peu de marge pour les services à la collectivité, service gratuit mettant en cause les compétences d'un universitaire. Jusqu'à maintenant, la confiance a toujours marqué la vie universitaire, le professeur déterminant les services à la collectivité qu'il considère essentiels dans sa charge de travail. Le ministre exige des moyens de vérifier non seulement la réalisation du travail, mais aussi la productivité et son imputation au budget de l'éducation.

Les propos du ministre et ceux du Conseil supérieur de l'éducation précisent que les services à la collectivité ne sont que des fonctions dérivées du rôle fondamental de l'université, soit la recherche et l'enseignement; cela laisse prévoir une réorganisation de la charge de travail des professeurs. Dans la mesure où le travail en comité; le travail administratif et syndical deviendront l'objet d'une remise en question, la charge de travail sera modifiée: on ne reconnaîtra, comme travail productif, que l'enseignement et la recherche.

Cependant, les effets des compressions budgétaires ne touchent pas que les services à la communauté. Le gouvernement actuel a prévu depuis longtemps une planification de la recherche au Québec. Or cette planification a des effets sur la vie universitaire et sur la charge de travail. Cette volonté s'est manifestée clairement dans le mandat de la Commission d'étude sur les universités. Comme le remarque le Conseil des universités dans son avis,

Les thèmes confiés aux trois comités de la Commission d'étude ont un caractère parcellaire. Le CEU avait à réussir le tour de force de définir une conception et les objectifs de développement pour l'université, milieu d'enseignement et de recherche, en ne traitant ni des programmes d'études de $2^{\mathrm{e}}$ et $3^{\mathrm{e}}$ cycles, ni de la recherche, ce dernier thème, on le sait, ayant été confié au groupe responsable du Livre vert gouvernemental pour une politique de la recherche. Dans son avis de 1977 sur le mandat de la CEU, le Conseil avait mis en doute la sagesse d'une telle décision, en soulignant que le fil conducteur des travaux de la Commission, 
dans un tel contexte, serait difficile à cerner. Il ne peut aujourd'hui que confirmer les propos tenus alors ${ }^{17}$.

En séparant la recherche du débat sur l'université, on permet l'étude de la productivité de la recherche, indépendamment du contexte de l'enseignement. Par le biais de la situation économique on oblige ainsi les professeurs-chercheurs à justifier leurs salaires en montrant la productivité de leur recherche. Le même raisonnement s'applique à la recherche comme aux services à la communauté. Les salaires investis sont-ils justifiés par les services rendus? En 1979, la Commission d'étude sur les universités avait déjà prévu les problèmes auxquels nous sommes confrontés aujourd'hui. Dans la deuxième partie du rapport du Comité d'étude sur l'organisation du système universitaire, on peut lire:

Mais une confusion survient lorsqu'on affirme quà peine plus de $20 \%$ des professeurs d'université font effectivement de la recherche. On se demande alors, si telle est la réalité, pourquoi fait-on toujours état de la double mission universitaire: enseignement et recherche? Ne devrait-on pas confier à d'autres agences que les universités les recherches jugées prioritaires par l'État ${ }^{18}$.

Dans la mesure où la recherche universitaire sera soumise à un contrôle extérieur, par le biais de la recherche subventionnée, les fonctions mêmes de l'université seront modifiées. Tel que prévu dans le mandat de la Commission d'étude, les $2^{\mathrm{e}}$ et $3^{\mathrm{e}}$ cycles sont déjà soumis aux politiques de la recherche et échappent ainsi à l'étude globale de l'université. Si, de plus, on enlève toute crédibilité à la recherche non subventionnée, ne risque-t-on pas de voir la charge de travail ramenée, pour les professeurs de $1^{\text {er }}$ cycle, à l'enseignement et l'encadrement des étudiants?

Les propos du ministre Laurin confirment cette approche, car ils proposent une réforme du $1^{\text {er }}$ cycle en vue d'un rendement supérieur: éviter le dédoublement inutile des enseignants et de la recherche par une planification de réseau, éviter aussi le dédoublement des enseignants entre le CEGEP et l'université, et assurer une meilleure adaptation de l'étudiant au marché du travail. Il suffit de relire la conférence du ministre Laurin pour se rendre compte qu'un diagnostic a été posé et qu'il n'existe pour lui qu'un seul remède.

17. Conseil des universités, op. cit., p. 18.

18. Commission d'étude sur les universités, Comité d'étude sur l'organisation du système universitaire, Rapport, Partie 2, p. 33. 
Considérer les activités du $1^{\text {er }}$ cycle, ce sera d'abord faire un certain tri parmi les activités existantes et les nouveaux projets, dans le but d'identifier et de choisir ce qui s'apparente le mieux aux finalités des institutions. Consolider, ce sera améliorer la qualité des activités d'enseignement de ce niveau, leurs fondements disciplinaires et méthodologiques, l'encadrement des étudiants, la pédagogie. Ce sera aussi rééquilibrer certaines programmation, de manière à tenir compte, selon que le $1^{\text {er }}$ cycle conduit au marché du travail ou aux cycles supérieurs, des exigences de la formation pratique et du degré de spécialisation qu'il importe de ne pas dépasser au $1^{\mathrm{er}}$ cycle.

Deuxièmement, il est impératif de stimuler le développement des études de $2^{\mathrm{e}}$ et $3^{\mathrm{e}}$ cycles et de la recherche ${ }^{19}$.

La proposition du ministre, en distinguant deux types de formation universitaire, le $1^{\text {er }}$ cycle et les $2^{\mathrm{e}}$ et $3^{\mathrm{e}}$ cycles, exige qu'on en précise les objectifs. Évidemment, les $2^{\mathrm{e}}$ et $3^{\mathrm{e}}$ cycles seront caractérisés par la recherche et s'intégreront, de ce fait, à la politique de la recherche. Ainsi, la planification de la recherche déterminera l'enseignement supérieur. On spécialisera, probablement, l'enseignement supérieur aux $2^{\mathrm{e}}$ et $3^{\mathrm{e}}$ cycles selon le champ de recherche reconnu à une université donnée, par la politique de la recherche. La planification de la recherche au niveau du réseau permettra à son tour, une planification des enseignements supérieurs. Selon cette hypothèse, les étudiants devront être mobiles puisque leur champ de spécialisation au $2^{\mathrm{e}}$ ou $3^{\mathrm{e}}$ cycle sera attribuée à une autre université. Cette politique conduira aussi à distinguer deux catégories de professeurs, ceux du $2^{\mathrm{e}}$ et $3^{\mathrm{e}}$ cycles, les professeurs-chercheurs et ceux du $1^{\text {er }}$ cycle, les professeurs; chaque catégorie ayant une charge de travail différente.

Puisque le premier cycle est détaché du $2^{\mathrm{e}}$ et $3^{\mathrm{e}}$ cycles, il doit être redéfini en fonction d'objectifs spécifiques. La solution proposée par le ministre, cherche à adapter l'université aux conditions actuelles qui exigent de la part des futurs travailleurs une mobilité accrue. Pour assurer cette mobilité, il faut dès lors réduire les barrières entre le CEGEP et le $1^{\mathrm{er}}$ cycle universitaire de même qu'assurer une mobilité entre les différents programmes du $1^{\mathrm{er}}$ cycle. Détaché ainsi des $2^{\mathrm{e}}$ et $3^{\mathrm{e}}$ cycles, l'enseignement au $1^{\mathrm{er}}$ cycle universitaire doit être défini par rapport à l'enseignement collégial. Présentement, on peut constater que les débats autour

19. Docteur Camille Laurin, op. cit., p. 9. 
de l'enseignement collégial ne sont pas terminés. Deux conceptions s'affrontent à ce niveau: définir le CEGEP comme prolongement des études secondaires en terme de formation générale ou le définir comme début des études post-secondaires en relation avec le premier cycle universitaire. Le choix entre l'une ou l'autre conception aura donc une influence considérable sur la définition des objectifs de l'enseignement universitaire au $1^{\text {er }}$ cycle. Les propos du ministre semblent prévilégier la seconde conception. Le système scolaire au Québec serait donc constitué de trois composantes: le primaire et le secondaire ayant pour objectif premier la formation générale, le CEGEP et le premier cycle universitaire conduisant au marché du travail, et les $2^{\mathrm{e}}$ et $3^{\mathrm{e}}$ cycles universitaires préparant les chercheurs.

Concevoir la formation universitaire au $1^{\text {er }}$ cycle et celle du CEGEP en termes de mobilité conduit inévitablement à prendre position sur la question de la spécialisation des enseignements car mobilité et spécialisation s'avèrent incompatibles. On reproche à l'enseignement universitaire d'être trop spécialisé dès le premier cycle. Cependant, ces reproches sont rarement appuyés d'une analyse. Ainsi s'explique l'apparition de plusieurs évaluations incompatibles justifiant le même jugement. Par exemple:

1. L'enseignement est trop spécialisé pour le marché du travail. À partir de critères de rentabilité, l'enseignement est jugé trop spécialisé s'il porte sur connaissances et des apprentissages qui ne sont pas immédiatement utilisables sur le marché du travail. On se demande à quoi cet enseignement peut servir plus tard.

2. L'enseignement est spécialisé trop exclusivement en fonction du marché du travail. Ceux qui formulent ce jugement contraire au précédent s'appuient sur les critères suivants: le manque de culture et/ou le manque d'esprit critique dans la formation universitaire. On reproche alors aux programmes d'enseignement du $1^{\text {er }}$ cycle de préparer des techniciens avec une information professionnelle ou disciplinaire et on déplore l'absence de formation intellectuelle permettant une distanciation et un jugement critique fondé. 
3. L'enseignement disciplinaire est trop spécialisé.

Cette fois la critique s'adresse à l'enseignement donné dans une discipline. Le problème consiste à cerner ce qui est essentiel à une discipline. Sur ce point les enseignants ne s'entendent pas. Pour certains, les secteurs de pointe dans leur discipline apparaissent comme l'essentiel, les autres secteurs tenant de l'érudition. Pour d'autres, au contraire, les résultats des recherches récentes n'ont pas encore fait leurs preuves, c'est pourquoi l'enseignement universitaire doit porter sur les acquis de la discipline.

Pour ne pas dépasser le degré de spécialisation du $1^{\mathrm{er}}$ cycle universitaire, le ministre propose le «retour à l'essentiel» dans l'esprit de la formation fondamentale du CEGEP (il ne faut pas confondre formation fondamentale et formation générale) qui consiste à donner un enseignement dont les contenus seront les fondements disciplinaires et méthodologiques. Cette orientation suppose qu'on puisse établir pour chaque discipline ce qui la caractérise en particulier malgré le contexte de l'éclatement des savoirs.

Le modèle comptable réduit l'accountability aux seules composantes de l'efficience et de la productivité, et intègre la dimension de l'efficacité à celle de la rentabilité par rapport au marché du travail. Le débat traditionnel sur les universités est donc transformé puisqu'il posait le problème en termes de valeurs culturelles et de formation intellectuelle, en termes non mesurables. Ainsi l'approche comptable permet d'éviter de longs débats sur les réformes à réaliser dans les universités, puisqu'elle renverse les données du problème. Alors que l'approche traditionnelle supposait qu'un accord sur les valeurs de l'enseignement justifiait les investissements concernés, l'approche comptable pose d'abord la question des investissements, cette dernière déterminant les valeurs sociales. Toute décision concernant l'université se fait dès lors sous le signe de l'économie (dans les deux sens du mot, i.e. planification budgétaire et «faire autant avec moins de ressources»), éliminant ainsi le débat sur la qualité des réformes en cause. De plus, dans la mesure où le modèle comptable est partagé par l'opinion publique, toute rhétorique touchant la qualité sera interprétée comme un refus de coopérer et comme une justification défensive des intérêts personnels. 


\section{CONCLUSIONS}

Il n'y a pas si longtemps, les revues philosophiques publiaient de nombreux articles sur la distinction entre les jugements de fait et les jugements de valeur. Certains philosophes avaient la «mauvaise habitude» de considérer les valeurs comme des objets en soi ou de prétendre qu'un jugement de valeur était susceptible de vérité ou de fausseté comme les jugements de fait. Cette distinction acquise, le discours sur les valeurs quitte le terrain de la démonstration certaine pour être soumis aux aléas de la rhétorique. La description précédente de l'évolution du débat sur les universités montre certains aspects de notre rhétorique québécoise sur l'université comme valeur sociale, valeur incontestée, valeur dépendante de la société, valeur comptable, et sur le rôle de cette thétorique dans la prise de décision. Mais, le problème théorique et pratique (car le discours est une pratique) subsiste. Si on ne peut pas démontrer la valeur certaine d'une chose, est-ce que tous les discours sur la valeur sont équivalents? $\mathrm{Ne}$ pouvons-nous pas toujours nous demander s'il existe une meilleure forme d'argumentation qu'une autre, évaluée selon des critères formels (auditoire universel) ou selon des critères d'efficacité?

Et si on conclut que l'efficacité est le critère premier pour évaluer l'argumentation ne devra-t-on pas admettre que le gouvernement actuel a les meilleurs arguments face à l'opinion publique?

Département de philosophie Université de Sherbrooke 